\title{
ESTUDO DE PADRÕES DE INTERAÇÃO PARA O DESIGN DA INTERFACE IIT
}

\author{
INTERACTION PATTERN STUDY FOR IIT INTERFACE DESIGN
}

Ravi Passos

\begin{abstract}
Artefatos de interação por toque são cada vez mais utilizados na sociedade contemporânea. O presente estudo tem por objetivo a proposição de uma gramática de toques para um projeto de design de interface interativa. A partir de análises paramétricas de artefatos com interfaces naturais e de levantamento documental, define-se um conjunto de toques determinado para utilização no projeto IIT.
\end{abstract}

Palavras chave: interação, interface, design

The use of touch-based interactive artifacts has grown exponentially in the contemporary society. The present study, aims to propose a grammar of touches for an interactive interface design project. Using a parametric analysis of natural interfaces artifacts and a documentary survey, the study results on a defined touchs set to be used in the IIT project.

Palavras chave: interaction, interface, design 


\section{Introdução}

A presente investigação trata do estudo da interação para o desenvolvimento de uma aplicação para o projeto IIT - acrônimo para Interface de Interação por Toque (Figura 1), o qual integra a parte prática da pesquisa "Design da Informação: um modelo para configuração de interface natural” [PASSOS, 2014]. Essa aplicação se dá por meio do desenvolvimento de uma "interface natural" do tipo multitoques e, para seu uso, se faz necessário um estudo sobre os padrões de interação a serem considerados no projeto do design de sua interface.

Interface Natural, também conhecida como Natural User Interface - NUI, é o termo usado para designar as interfaces digitais interativas que utilizam linguagens naturais para seu uso. Este tipo de interface tem sua interação mediada por voz, visão, gesto, toque, tato, escrita manual, dados biométricos, distinção facial, assim como pelos demais estímulos sensório-motores intrínsecos ao homem.

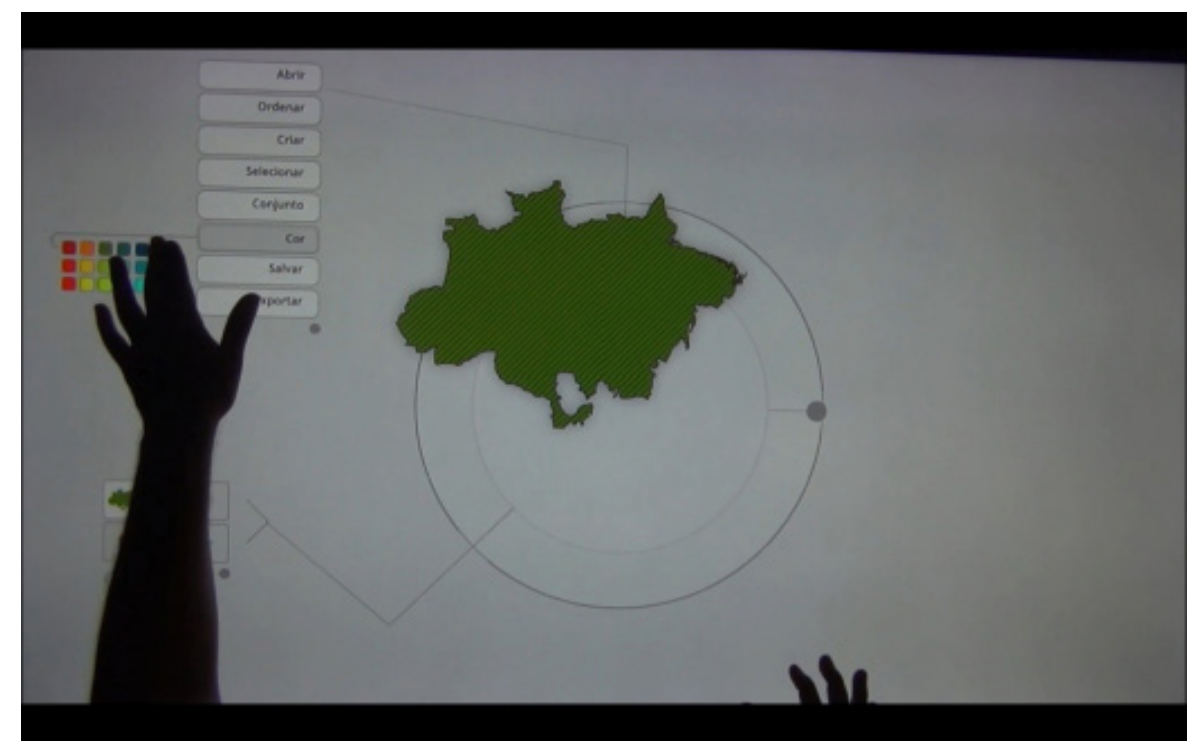

Figura 1: Frame do filme de apresentação da IIT.

Em levantamento executado em Passos [2014] aferiu-se que o tema ainda é restrito no que tange conceitos e definições, e que, segundo Norman, [2010] a nomenclatura - interfaces naturais - tem forte relação com fatores de mercado e de marketing, estando à frente da realidade em que estes artefatos se encontram em relação à sua potencialidade de uso. Portanto, se infere primeiramente que é necessário um grande avanço em relação ao desenvolvimento deste tipo de interface, e ainda, que as regras e modelos utilizados para as interfaces tradicionais ainda são pertinentes ao contexto das atuais interfaces ditas naturais. Ainda assim, entende-se que o termo delimita um conjunto determinado de artefatos e, por esse motivo, mantém-se seu uso.

Ainda em Norman [2010], discute-se que o desenvolvimento deste tipo de interface não é recente, encontrando-se iniciativas na literatura, como o Apple Newton em 1992, que teve seus estudos iniciados na década de 1960; pesquisas sobre realidade artificial realizados por Myron Kruger, anteriores à década de 1980; e um sistema multitoques descrito na dissertação de Nimish Mehta, em 1982. Além dessas iniciativas, o autor explica que sensores de movimentos e localização humana, sintetizadores e instrumentos musicais multitoques também já existem a um tempo considerável. 
Entre os vários tipos de interfaces naturais existentes, observa-se de maneira notória, um que está em avançado nível de desenvolvimento e utilização em artefatos comerciais como tablets e smartphones: as interfaces que têm como paradigma de interação o uso de multitoques.

Tanto o paradigma de interação quanto a tecnologia de interação por multitoques possibilitam o desenvolvimento de um conceito aplicado a artefatos, que têm agora sua interação com os usuários de uma maneira distinta, sobrepondo-se à questão da necessidade do uso de periféricos, renovando o contexto de interação. [OLIVEIRA; SILVA, 2008].

Isso implica em uma interação direta do usuário com a interface, por meio de uma gama de toques possíveis, o que reestabelece o conceito de 'manipulação direta' desenvolvido por Douglas Engelbart no Xerox Parc - Palo Alto, dispensando o uso de periféricos de entrada como mouse e teclado para mediar a interação.

Segundo Preece et al [2005], um paradigma de interação é uma filosofia ou uma maneira específica de se pensar interação, e pode colaborar na orientação do projeto de design. Durante muitos anos o paradigma de interação que prevaleceu em artefatos digitais foi o desenvolvimento de aplicações para desktops (computadores de mesa), vinculando sua utilização a um indivíduo sentado em frente ao monitor, CPU, mouse e teclado, e acessando um sistema por meio de uma Interface Gráfica do Usuário (Graphic User Interface - GUI).

As interfaces naturais, como as apresentadas ao grande público em filmes de ficção há muitos anos, já têm versões similares em desenvolvimento acentuado, sendo algumas já disponibilizadas comercialmente em formatos de artefatos diversos, principalmente mediados por toques e gestos.

Conforme apresentado a seguir, existe um crescente desenvolvimento deste tipo de interface - que não utiliza uma tela tradicional, tampouco utilizam os convencionais mouse e teclado para mediar a interação entre usuário e informações -, intencionando propiciar a interação mais fluída. Podem ser citados brevemente como exemplos de artefatos que utilizam interfaces naturais, os smartphones, que funcionam com

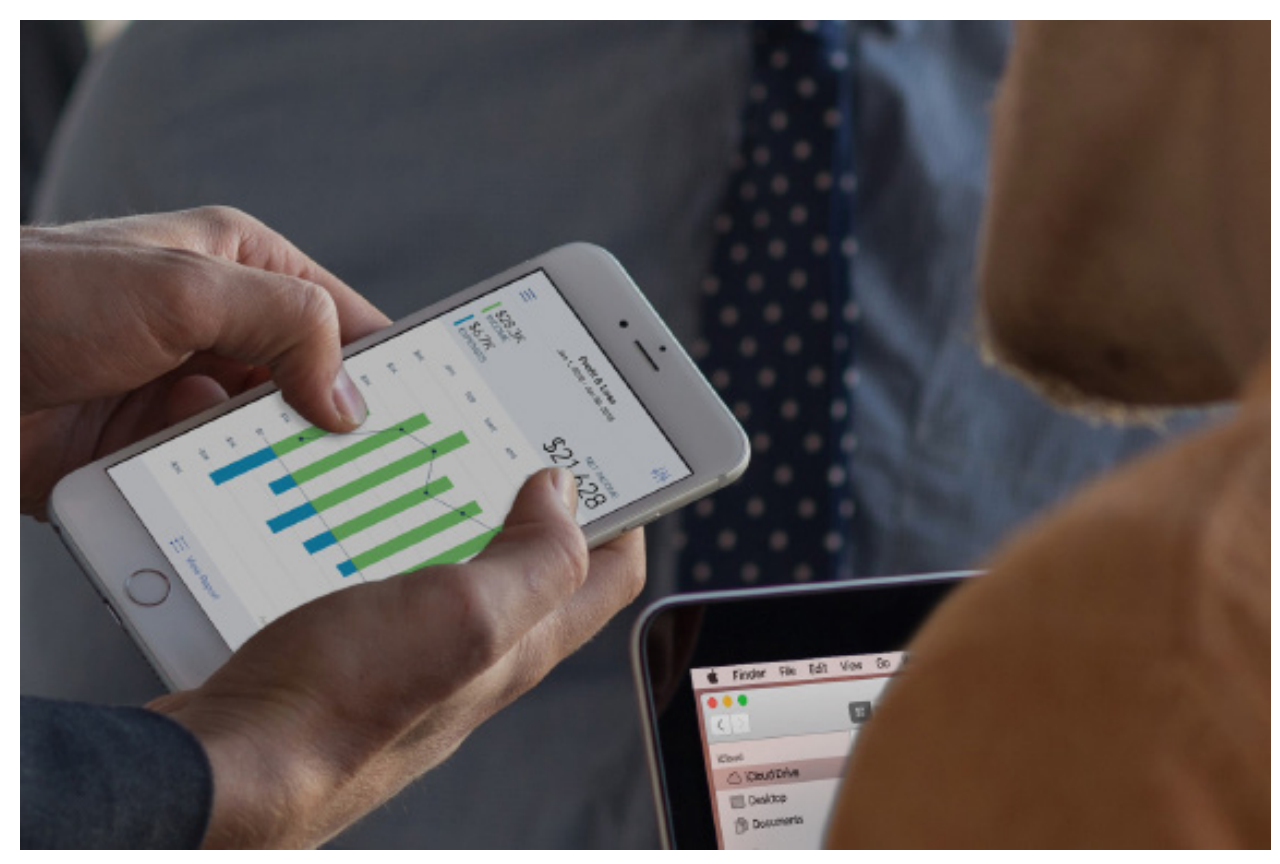

Figura 2: Exemplo de artefato (iPhone) que utiliza interação por multitoques.

Fonte: <http://www.apple.com/br/iphone/>. Acesso em: 07/05/2017. 
tela de multitoques (Figura 2).

Como etapa do percurso metodológico de desenvolvimento do trabalho, entende-se ser importante a realização de um levantamento sobre artefatos contemporâneos relacionados às interfaces naturais, em vista da compreensão do argumento de Norman [2010], quando coloca em cheque o real desenvolvimento desse tipo de interfaces (e a nomenclatura utilizada). Esse levantamento também possibilita o conhecimento de características e parâmetros que esclareçam o que são e como se constituem as ditas "interfaces naturais" atualmente em desenvolvimento. Tal atividade possibilita a tomada de partido a respeito do uso desse tipo de interfaces para o trabalho em questão, e assim, permite uma fundamentação para a proposição de uma gramática específica para a interação no projeto IIT.

Para além das iniciativas apresentadas por Norman [2010], o estudo e desenvolvimento de artefatos com interface natural, no que tange hardwares e interfaces com interação por toque, as quais são acessíveis ao grande público e a indústria de massa, é algo relativamente recente.

Entre o final da década de 1990 e começo de 2000, telas que permitem interação por toque iniciaram seu desenvolvimento até o uso do grande público, por meio de quiosques de venda, telas de informação ao público, serviços de check-in em aeroportos, sistemas de bilhetes de transporte e terminais eletrônicos de autoatendimento bancário. [SAFFER, 2009]

Neste cenário, a tecnologia mostrou-se um elemento de grande importância, pois permitiu a superação de potenciais de uso de artefatos interativos de maneira acelerada. Desse modo, os estudos contemporâneos demandam reflexões e atualizações intensas e, o presente estudo, se justifica pela necessidade de reflexão sobre a evolução tecnológica, aliada ao desenvolvimento teórico e metodológico na área do design e seus

assuntos correlatos (interface, interação, acessibilidade, etc.).

\section{Caracterização do projeto IIT}

O projeto IIT caracteriza-se pelo desenvolvimento de uma interface natural que usa o paradigma de interação denominado multitoques. Esta interface se

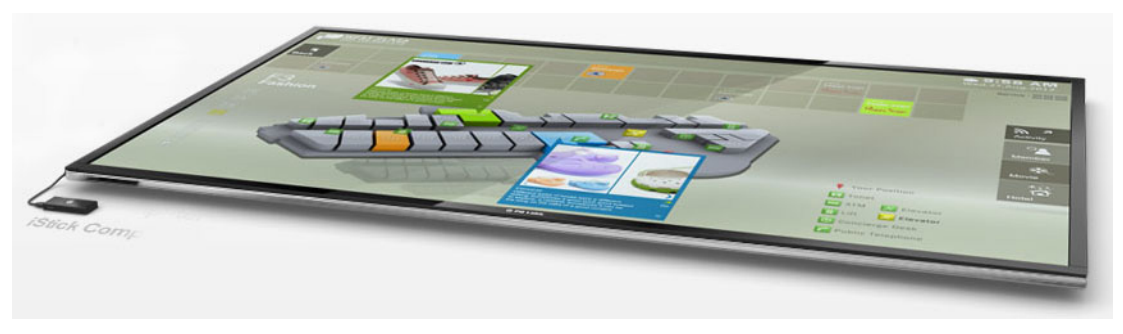

Figura 3: Hardware estipulado para o projeto da IIT: PQ Labs Multi-Touch overlay. Fonte: $<$ http://multitouch.com/product.html $>$. Acesso em: 14/o8/2013

destina à mediação entre o usuário e um aplicativo de tratamento e manipulação de informações de Sistemas de Informação Georreferenciadas - SIG. A IIT tem como acesso à sua interface uma mesa de multitoques de grande formato constituída a partir do equipamento desenvolvido pela PQLabs (Figura 3).

A abordagem metodológica utilizada no processo
[PASSOS, 2008a, 2008b, 2014] tem caráter cartesiano para seu desenvolvimento e o presente estudo propõe-se a atuar em um ponto determinado, fundamental à sua realização: os padrões de interação necessários para seu uso, que constituem uma gramática específica.

Os usuários da IIT são especialistas no tratamento 
gráfico de informações destinadas a utilização por sujeitos não especialistas, para a tomada de decisão, tais como, funcionários públicos de órgãos de fiscalização de recursos naturais de instituições governamentais de gestão ambiental do Brasil, que necessitam gerar relatórios de monitoramento e planos de ações baseados no estado em que se encontram os territórios e seus atributos.

Outra característica sobre os usuários da IIT é que esses não são necessariamente especialistas em informações georreferenciadas - no que tange a concepção e edição dessas informações -, mas podem consultá-las para obter insumos na realização de suas atividades (ex.: uso de imagens em relatórios). Sendo assim, a IIT privilegia a etapa específica de geração de imagens.

É importante frisar que a atividade de geração de imagens na IIT está no âmbito dos SIG. Tais sistemas são utilizados para o tratamento e manipulação de informação para usos diversos, possibilitando, em instâncias finais, a geração de arquivos de fins secundários, a tomada de decisões por autarquias administrativas e a geração de insumos para relatórios técnicos.

A definição da tarefa a ser realizada no projeto foi determinada por especialistas a partir da utilização do programa ArcMap, componente do ArcGIS Suite, tendo sido descrita toda a atividade relativa à tarefa designada para o projeto deste estudo, conforme Passos [2014]. Para o contexto deste trabalho, foi selecionada um conjunto de atividades específicas e representativas para o contexto do usuário e do objetivo da tarefa, respeitando tanto os objetivos da investigação como as restrições de seu escopo.

É ainda pertinente esclarecer que a definição da tarefa se fundamentou em estudos ergonômicos [ISO 9241, 1993; Nielsen e Molich, 1990; Bastien e Scapin, 1993; Cybis, 2003; Dix et al, 2004] e, aliado ao método de caso de uso, sendo estabelecido, de modo sistemático, o processo de uso pelo usuário da interface, apresentado na .

\section{Levantamento e análise de artefatos}

Tendo em vista a necessidade de definição de padrões de interação para o projeto IIT, se fazem necessários o levantamento e a análise de artefatos com interfaces naturais, na medida em que tais procedimentos possibilitam uma compreensão sobre este tipo de interface e suas interações. Além disso, tais procedimentos aproximam o entendimento global em relação às particularidades necessárias a uma interface multitoques de grande formato, tal como intencionado pelo estudo. Tanto os hardwares, levantados em vista da verificação de possibilidades reais de desenvolvimento, quanto as interfaces, levantadas em vista da definição de suas

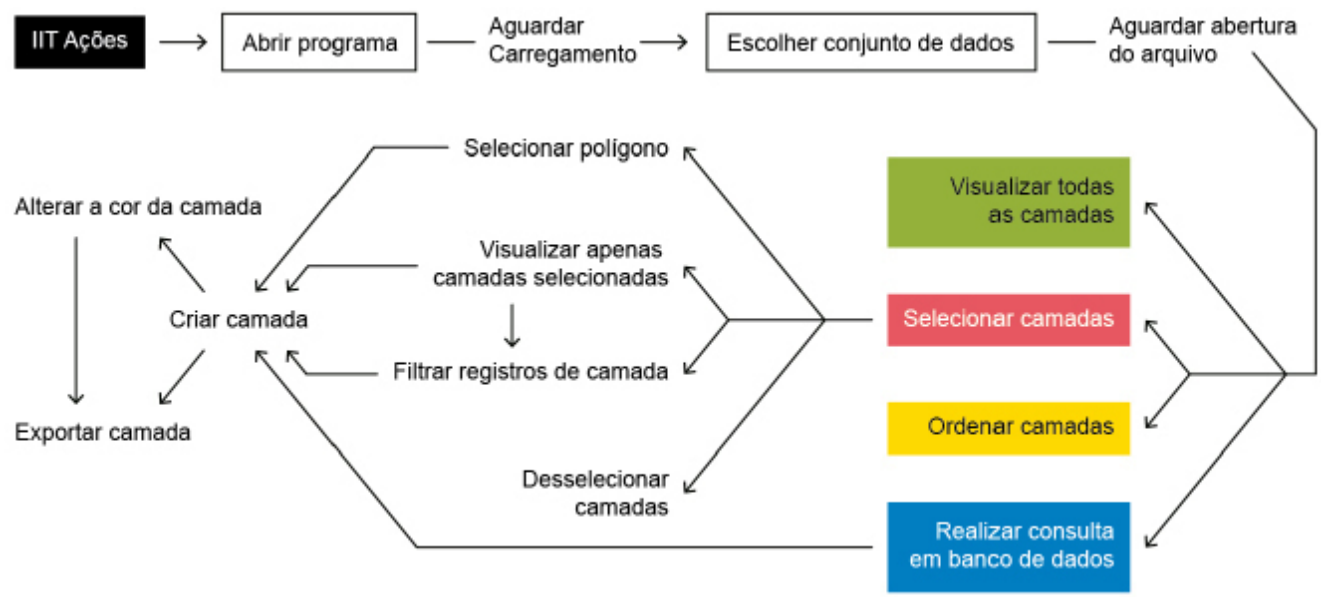

Figura 3: Fluxo de ações possíveis para interação entre usuário e IIT. 
relações de configuração em contexto de interação por multitoques, colaboram na construção técnica e idearia da interface natural do tipo multitoques, artefato utilizado como objeto de aplicação para o presente estudo, e que traz de modo intrínseco uma definição de sua gramática de toques específica. Existem inúmeros artefatos que têm as chamadas "interfaces naturais", entretanto, em conformidade com o escopo deste estudo, são delimitadas analises de artefatos que tenham interfaces naturais do tipo multitoques. Quando pertinente, de modo complementar e colaborativo, são tratados alguns artefatos e interfaces com interação por gestos, pois os mesmos têm relações de usos familiares à interação por toques, seja no modo de operação ou no resultado da ação.

O levantamento e a análise de artefatos que utilizam toques e gestos, apresentados a seguir, utilizam a ferramenta denominada 'análise paramétrica', pois a mesma possibilita a coleta e análise de parâmetros de maneira sistemática entre os diversos artefatos pesquisados. Segundo Baxter [1998], a análise paramétrica serve para comparar produtos a partir de certas variáveis chamadas de 'parâmetros comparativos', podendo ser utilizada para análises de aspectos quantitativos, qualitativos ou classificatórios.
No caso do presente estudo, são abordados dois tipos de análises: 'análise paramétrica de interface' (Tabela 1), que trata de interfaces digitais de caráter gráfico e 'análise paramétrica de hardware' (Tabela 2), que trata de equipamentos diversos interconectados às interfaces. A primeira análise se justifica, pois, como discutido, é no âmbito da interface do artefato que ocorre a configuração do conteúdo informacional, que é utilizado na interação. A segunda análise se justifica na medida em que é o hardware que traz suporte à interface, o que envolve potenciais e limitações, incluindo a maneira como ocorre a interação.

A 'análise paramétrica de interface' compreende o parâmetro 'interface' que é determinado pelas teorias estabelecidas no referencial teórico do projeto [PASSOS, 2014] relativo à composição da informação, no que tange seu conteúdo e conferindo a ele configuração determinada. Desse modo, as variáveis definidas para este parâmetro são determinadas, em relação ao arranjo entre elementos de interface, por meio do grid, assim como de seus elementos imagéticos (grafismos e ícones) e textuais (tipografia). O 'sistema operacional' utilizado pela interface também é utilizado como parâmetro dessa análise paramétrica, pois a existência de recor-

\begin{tabular}{lll} 
Parâmetro & variável & Característica do artefato \\
& Grafismos & Caracterização de grafismos \\
\cline { 2 - 3 } Interface & Tipografia & Utilização de serifa \\
\cline { 2 - 3 } & Grid & Tipo de grid \\
\cline { 2 - 3 } & Ícones & Características iconográficas \\
\hline Sistema Operacional & Tipo & Tipo de sistema operacional \\
\hline
\end{tabular}

Tabela 1: Parâmetros e variáveis da 'análise paramétrica de interface'.

rência no uso de determinados tipos de sistemas operacionais pode implicar na existência de similaridade em características visuais.

Portanto, os parâmetros e as variáveis analisados na 'análise paramétrica de interface' são:
De modo complementar, e com importância secundária em relação à 'análise paramétrica de interface', realizou-se uma 'análise paramétrica de hardware', que tem como parâmetro a 'interação' propiciada por ele, pois este é um elemento determinante nas interfaces naturais. Como variável do 
parâmetro ‘interação’, observa-se a necessidade de utilização de 'acessórios' pelo hardware, o que influencia sobremaneira o modo de interação utilizado. Ainda como variável, observa-se a quantidade de 'usuários' simultâneos e de 'pontos de toques ativos' simultaneamente, que influenciam igual- mente na interação. Como parâmetro, verifica-se, adicionalmente, a 'tecnologia' utilizada pelo hard-

\begin{tabular}{lll} 
Parâmetro & variável & Característica do artefato \\
\cline { 2 - 3 } Tnteração & Acessórios & Paradigma utilizado \\
\cline { 2 - 3 } & Quantidade de usuários & Túmero de usuários simultâneos \\
\cline { 2 - 3 } & Pontos de toque & Quantidade de toques simultâneos \\
\hline Tecnologia & Tipo & Modo de captura da interação \\
\hline Dispositivo & Tamanho & Tamanho da área de interação \\
\hline
\end{tabular}

Tabela 2: Parâmetros e variáveis da 'análise paramétrica de hardware'.

ware com variável 'tipo', afim de se compreender como é capturada a interação pelo sistema. Por fim, utiliza-se o parâmetro 'dispositivo' com a variável 'tamanho', que novamente influencia a interação. Desse modo, os parâmetros e as variáveis analisados na 'análise paramétrica de hardware' são:

A análise paramétrica proposta foi estruturada a partir de uma pesquisa exploratória com uma amostra limitada, mas que permite a compreensão global sobre artefatos que utilizam toques e gestos como paradigma de interação. Esse levantamento reuniu desenvolvimentos de artefatos de natureza comercial e pesquisas acadêmicas. Foram analisados os seguintes artefatos:

1. Perceptive Pixel;

2. Microsoft Surface;

3. NUITEQ;

4. Intuiface Presentation;

5. Microsoft Kinect Sensor;

6. Nintendo Wii;

7. PlayStation Move;

8. MYO;
9. Oblong - Mezzanine;

10. Evoluce Win\&I;

11. Fraunhofer FIT;

12. Leap Motion;

13. Samsung Smart TV;

14. Grasshopper Canvas with Kinect Interaction;

15. Sixthsense;

16. SoundWave;

17. Softkinetic;

18. Digits Bracelet;

19. Hitachi Canesta TV;

20. PQ Labs Multi-touch.

Cabe ainda mencionar que as informações obtidas foram limitadas pelas condições e possibilidades do contexto deste estudo e dos artefatos pesquisados, o que reflete, eventualmente, em certas restrições de aprofundamento, pois esses levantamentos são diretamente relacionados às informações disponibilizadas pelas empresas ou instituições responsáveis por cada projeto. 
O levantamento contou com uma coleta de artefatos em diferentes estágios de desenvolvimento finalizados, conceituais ou em estudo -, visando o entendimento de suas características individuais. Verificou-se que os artefatos em desenvolvi- mento, geralmente, não dispõem de informações aprofundadas para suas análises, mas ainda assim considerou-se positiva a inclusão dos mesmos na pesquisa, na medida em que se objetiva o aprofun-

\begin{tabular}{lll} 
Parâmetro & variável & Características dos artefatos mais observadas* \\
Grafismo & $\begin{array}{l}\text { Elementos retangulares, elementos ortogonais, formas } \\
\text { geométricas, cores sólidas }\end{array}$ \\
\cline { 2 - 3 } Tipografia & Fonte sem serifa \\
\cline { 2 - 3 } & Grid & Modular e hierárquico \\
\hline Ícones & Geométricos, monocromáticos, com legendas \\
\hline Sistema operacional & Tipo & Microsoft Windows e sistema próprio \\
\hline
\end{tabular}

* Foram omitidos resultados não se aplica mesmo que em grande quantidade, pois são referentes a artefatos que não possuem interface própria.

Tabela 3: Resultado da análise paramétrica de interface.

damento do conhecimento sobre o tema.

A seguir, apresenta-se uma sistematização dos levantamentos com as informações relevantes para o contexto deste estudo e que se sobressaíram em relação à recorrência (Tabela 3 e Tabela 4).

Verifica-se na análise paramétrica de interfaces que, de modo geral, as interfaces utilizam grafismos geométricos, com utilização de elementos ortogonais, que tendem para formas retangulares e que utilizam cores sólidas. Tais características se assemelham ao estilo Flat, recorrente em interfaces digitais interativas contemporâneas. Isso reforça a inferência inicial de que as interfaces naturais se utilizam de parâmetros utilizados por interfaces tradicionais.

Também se entende que a simplicidade, predominante nas composições, evidencia uma preocupação nas questões de usabilidade, uma vez que a manipulação de elementos de modo direto e pontual na interface exige cuidados significativos, se con-

\begin{tabular}{lll}
\multirow{2}{*}{ Parâmetro } & variável & Características dos artefatos mais observadas \\
\cline { 2 - 3 } Interação & Acessório & Multitoques e gestos \\
\cline { 2 - 3 } & Quantidade de usuários & Individual e múltiplos \\
\cline { 2 - 3 } & Pontas e toque & Não descrito ou não se aplica \\
\hline Tecnologia & Tipo & Tela sensível a toques, câmera, sensor IR \\
\hline Dispositivo & Tamanho & 55 e 82 polegadas e não se aplica \\
\hline
\end{tabular}

Tabela 4: Resultado da análise paramétrica de hardware. 
siderado o modo relativamente novo de interação, cabendo a compreensão de que há a necessidade de aprendizagem por parte do usuário.

Em relação à análise de hardware, verifica-se que a maior parte dos artefatos utiliza a captura da ação do usuário, sejam toques ou gestos, por sensores. Algumas análises mostram, ainda, a utilização de artefatos de manejo que, geralmente, possuem sensores de comunicação entre as partes do hardware, o que direciona sua classificação para um tipo de artefato híbrido entre interfaces tradicionais, devido à necessidade de dispositivos de entrada e interfaces naturais, pois permitem uma interação mais relacionada aos movimentos corporais do usuário.

Em relação à área de interação, foi priorizado no levantamento, o uso de interfaces de toque de grande formato, que por sua vez permitem interações de múltiplos usuários com múltiplos toques. Considerando uma questão tecnológica, tal delimitação influencia determinantemente na proposta desse estudo, pois cada usuário pode efetuar um número determinado de toques durante a interação que pode duplicar a cada usuário participante da interação, sendo que os artefatos disponíveis possibilitam um número determinado de toques simultâneos.

Os levantamentos realizados foram relevantes para o conhecimento deste tipo de artefato - que utilizam interfaces naturais -, e de seus atributos. Também é possível, a partir das análises paramétricas realizadas, observar parâmetros congruentes com o conceito de interface natural apresentado no inicio do trabalho.

Nota-se ainda que os artefatos pesquisados compreendem, em sua maioria, padrões de interações distintos dos propostos para interfaces tradicionais, apesar de manterem muitas relações de configuração entre si. Entende-se assim, que as afirmações de Norman [2010] são pertinentes, de certo modo, pois potencialmente, o conceito de interface natural pode abarcar uma quantidade muito superior de interações naturais, relativa aos sentidos humanos, como por exemplo utilizado por Nicolelis [2011], que atua na neurociência, área de desenvolvimento distinta do design e do tipo de interface interativa mencionada nessa discussão, e que não permite firmar um paralelo acessível a este estudo devido a discrepância de escopo.

\section{Padrões de interação}

Em complemento à análise sobre os artefatos de interface natural apresentada, também se faz pertinente um levantamento sobre a maneira de in- teração com este tipo de artefato, especificando como ocorre a ação de toques e gestos por parte do usuário. Assim, o levantamento realizado se dividiu em três etapas:

- Primeira etapa: reúne um apanhado sobre documentações referenciais de dispositivos com interação por toques e gestos;

- Segunda etapa: trata da exibição, por meio de amostragem, dealgumas dasinteraçõesutilizadas em artefatos da análise paramétrica efetuada, que se mostraram representativos em relação às ações de interação, complementarmente à primeira etapa;

- Terceira etapa: traz referências visuais de como são representados graficamente os toques e gestos, também intencionando uma melhor compreensão sobre o modo de interação utilizado em seu uso na comunicação de estudos obtidos.

A importância deste levantamento se acentua na medida em que favorece o conhecimento dos tipos de toques e gestos utilizados neste tipo de interface. Assim, torna-se possível a definição fundamentada de conjuntos de toques em situações específicas, como requerido por essa investigação, que conta com um ensaio pragmático da interface IIT, e consequentemente demanda a composição de uma gramática particular para si. Além disso, possibilita-se uma padronização de nomenclatura e classificação de cada tipo de movimento.

O levantamento realizado apresenta convenções adotadas por grandes empresas acerca do uso de toques e gestos suportados por seus produtos. Foram coletadas documentações de suporte ao desenvolvimento de aplicativos para Android [ANDROID DEVELOPERS, 2013] e IOS [IOS DEV CENTER, 2012], o Guia de Usuários do dispositivo Microsoft Surface [MICROSOFT SURFACE, 2013], além do Guia de Programação do Windows Touch [WINDOWS TOUCH, 2013]. Esses documentos possibilitaram uma definição global de interações no contexto de interfaces de multitoques.

Também foram consideradas no levantamento, as pesquisas de dois grupos de pesquisa na área, com ênfase em usabilidade e Interação Humano-Computador - IHC: o TouchThinking e LukeW Ideation \& Design. Vale uma ressalva qualitativa ao documento Touch Gesture Reference Guide [VILLAMOR et al, 2010], que trata de um apanhado sobre gestos de dispositivos móveis disponíveis ao grande público: o Android, o iOS e Windows Phone.

Como resultado do levantamento realizado, segue a Tabela 5, que dispõe os movimentos identificados, 


\begin{tabular}{|c|c|c|}
\hline Toque e gesto & Descrição & $\begin{array}{l}\text { Número de } \\
\text { ocorrências }\end{array}$ \\
\hline Deslizar & $\begin{array}{l}\text { Mover a mão, dedo ou dedos em uma direção para mover objeto ou } \\
\text { visualização }\end{array}$ & 9 \\
\hline Pinçar (abrir e fechar) & $\begin{array}{l}\text { Afastar ou aproximar mão ou dedos para redimensionar visualização } \\
\text { ou objeto }\end{array}$ & 9 \\
\hline Pivotar & $\begin{array}{l}\text { Mover mãos ou dedos em trajeto circular para rotacionar objeto ou } \\
\text { visualização }\end{array}$ & 7 \\
\hline Pressionar & $\begin{array}{l}\text { Selecionar objeto e manter o gesto usado na seleção por alguns segun- } \\
\text { dos para ativar outras opções }\end{array}$ & 5 \\
\hline Avançar & Mover a mão ou dedo na direção da tela sobre o objeto desejado & 4 \\
\hline Tocar & Tocar com a ponta do dedo na tela sobre o objeto desejado & 4 \\
\hline Espremer & $\begin{array}{l}\text { Mover a mão aberta na direção da tela sobre o objeto desejado e fechar } \\
\text { a mão }\end{array}$ & 3 \\
\hline Lançar & $\begin{array}{l}\text { Tocar com a ponta do dedo na tela, mover e levantar o dedo em um } \\
\text { movimento rápido }\end{array}$ & 3 \\
\hline Toque duplo & $\begin{array}{l}\text { Tocar com a ponta do dedo na tela sobre o objeto desejado duas vezes } \\
\text { em rápida sucessão }\end{array}$ & 3 \\
\hline Arrastar & Mover a mão ou dedo após selecionar objeto para reposicionar o objeto & 2 \\
\hline Girar & Mover uma mão ou dedo em trajeto circular & 2 \\
\hline Agitar & Agitar o dispositivo & 1 \\
\hline Avançar e pinçar & $\begin{array}{l}\text { Mover mão em direção a tela, avançar dois dedos em direção a tela e } \\
\text { aproximar os dedos entre si }\end{array}$ & 1 \\
\hline Pivotar para fora & $\begin{array}{l}\text { Tocar na tela com todos os dedos da mão próximos uns dos outros e } \\
\text { afastá-los }\end{array}$ & 1 \\
\hline Pressionar e tocar & Mover mãos ou dedos em trajeto circular para fora da área da tela & 1 \\
\hline Tocar em dois pontos & Tocar na tela em dois pontos simultaneamente & 1 \\
\hline
\end{tabular}

Tabela 5: Toques e gestos identificados por amostragem em vários dispositivos e sistemas; quantidade de ocorrências de cada toque ou gesto.

organizando-os do maior para o menor número de ocorrências de movimentos de interação por toques e gestos funcionalmente idênticos, entre os vários sistemas analisados.
Por fim, a Tabela 6 apresenta o produto da "terceira etapa", descrita anteriormente, e organizada de modo a comparar a maneira como acontece a representação visual dos gráficos que utilizam toques e gestos em suas documentações, conforme segue: Abstrata 


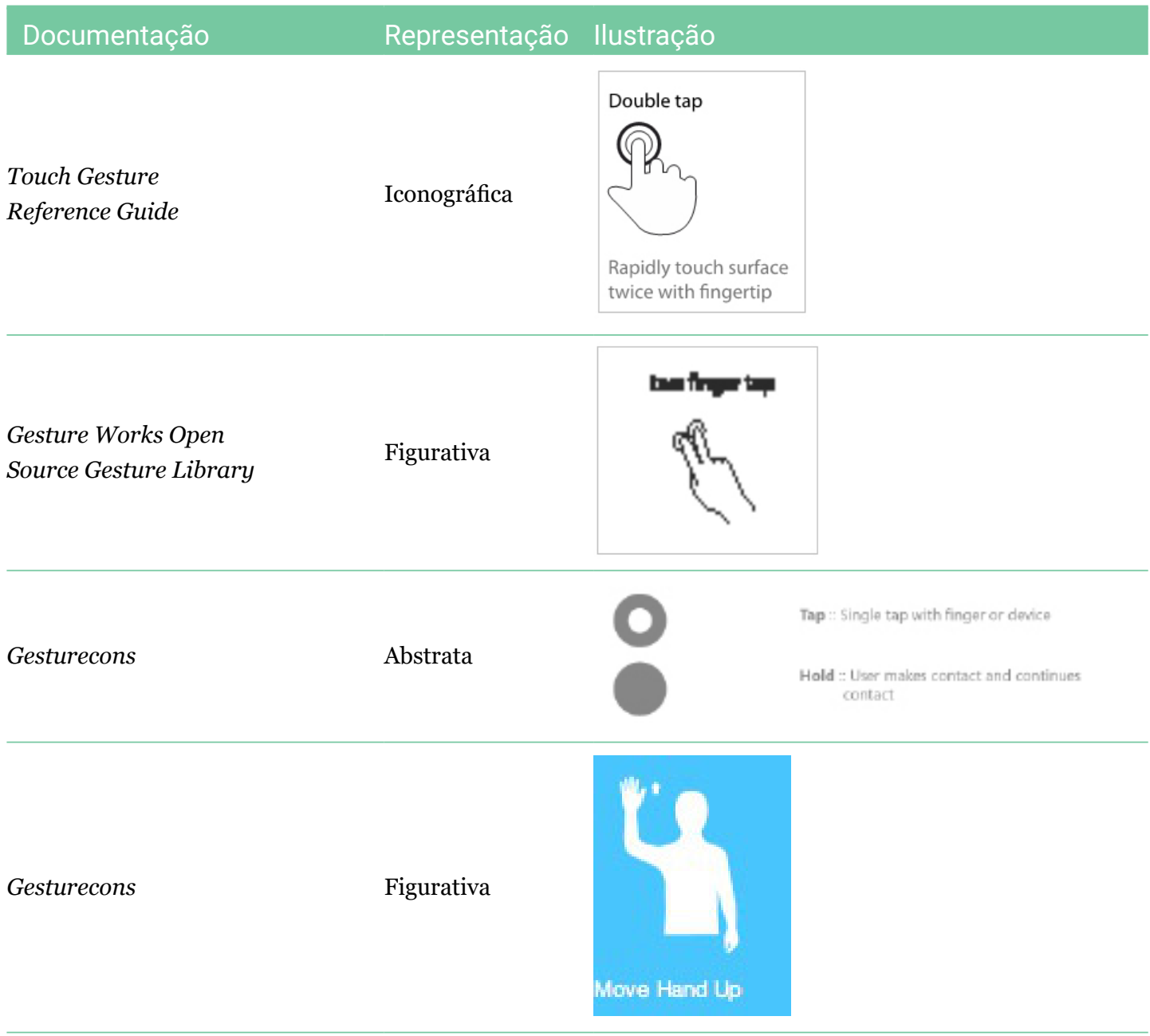

Tabela 6: Comparativo de representação de toques e gestos entre as documentações.

Observa-se a utilização de grafismos que variam entre representações figurativas e abstratas. Consideram-se figurativas as representações que têm maior detalhamento em relação aos objetos da natureza, enquanto abstratas, as representações aferidas de modo simbólico (ou simplificações de objetos da natureza). Encontram-se ainda, como elemento intermediário, as representações iconográficas que, apesar de uma fundamentação figurativa em sua forma, não detêm um alto nível de detalhamento.

Sistematicamente, em relação às propriedades que fundamentaram as necessidades de definição de representação gráfica, citam-se: acuidade, facilidade e rapidez de interpretação simbólica, ca- pacidade de redução na aplicação, possibilidade de aplicação em diferentes partes da interface e padronização de um estilo que englobe o conjunto das representações, considerando todos os fatores anteriores. Ainda são considerados na definição, as análises realizadas, que resultam na seleção de elementos mais utilizados nas diferentes plataformas.

Desse modo, considerando o contexto da demanda e das análises realizadas, entende-se que a representação gráfica mais eficiente ao presente estudo é a iconográfica. Além dos atributos supracitados, tal definição se fundamenta no resultado das análises paramétricas realizadas, que mostraram as ações nas interações e sua representação.

É interessante salientar que as representações figurativas encontradas, além de causarem eventuais polissemias, também possibilitam maior sobrecarga cognitiva ao usuário. Já as representações abstratas, por vez, requerem um nível de abstração 
do usuário e facilmente remetem a uma polissemia e maior sobrecarga cognitiva, podendo ser incompreensíveis, eventualmente.

\section{Delimitação do paradigma de interação}

Conforme delimitado nos objetos deste estudo, foi definido como paradigma de interação, o uso de multitoques. A associação das interfaces multitoques ao contexto dos SIG apresenta uma convergência de potenciais, que possibilita refinamento das questões de usabilidade, colaborando assim, no cumprimento das tarefas pré-definidas, e possibilitando a consolidação deste paradigma de interação de modo mais intuitivo e eficaz, e ainda, proporcionando melhor compreensão e distinção do conjunto do conteúdo, de seus elementos e de suas relações.

Em termos práticos, o estudo permite afirmar que a interação de SIG mediada por interfaces multitoques possibilita a realização da atividade de maneira mais eficiente, colaborando na interação do usuário com a informação de modo mais específico. Favorece ainda, a recuperação, a consulta e a comparação de informações diversas, além da produção e articulação

Poque Simples

de conteúdos e extração de insumos como gráficos, utilização deste padrão de interação pode favorecer tanto a compreensão de informação quanto a tomada de decisões estratégicas, e consequente transformação destas em ações efetivas.

Para a interface em questão, a IIT, foi necessária a definição de uma gramática padrão de toques possíveis para sua interação. As características usadas na definição iconográfica e o tipo de movimento foram fundamentados na recorrência aferida no estudo, na simplicidade de compreensão da ação e de ação requerida na atividade e o significado encontrado em cada ação/movimento.

A estratégia de associação entre a definição de representação (iconográfica) e a definição das ações (de interação) se mostrou interessante ao trabalho. Isso porque, inicialmente, possibilitou-se maior facilidade de comunicação, devido ao melhor entendimento de cada gesto a partir de sua representação, e com isso, uma melhor associação de cada uma das ações à tarefa pré-definida para a IIT.

A compreensão da tarefa relativa ao uso de SIG, em particular ao estipulado para a IIT, associada às possibilidades de multitoques levantadas, possibilita a definição das ações de interação, sendo textos, padrões, entre outros. Em termos gerais, a seu consecutivo uso, além da possibilidade entre a

Tabela 7: Conjunto de gestos estipulados para a IIT. 
elas: acionar, marcar, ligar, desligar, selecionar, arrastar, mover, ampliar, reduzir, aproximar, rotacionar, desenhar (polígonos) e escrever. Assim, foram definidas as seguintes ações a compor a gramática de interação da IIT: toque simples, pressionar, deslizar, pinçar, rotacionar e arrastar (Tabela 7).

Entende-se que o conjunto proposto permite ao usuário da IIT uma interação de grande usabilidade, contando com uma curva de aprendizagem satisfatória - objetivo do presente estudo. Do mesmo modo, a representação da gramática de interação também permite sua compreensão de maneira eficiente.

\section{Considerações finais}

Como visto, o projeto IIT trata do desenvolvimento de uma interface natural que utiliza como paradigma de interação o multitoques. Considerando uma abordagem metodológica cartesiana abrangente para seu desenvolvimento, o presente projeto propôs-se a estudar uma parte pontual e específica de tal abordagem: os padrões de interação necessários ao seu uso.

A partir de requisitos determinados para o projeto, foram delimitados instrumentos de análises comparativas de artefatos de interação em interfaces naturais, tanto de toques como de gestos, sendo este segundo paradigma, complementar.

Com o intuito de se determinar uma gramática de toques aderente às necessidades do usuário da IIT, destacam-se nesta atividade, tanto análises empíricas como levantamentos documentais. Como visto, vale ressaltar que a IIT é um instrumento para elaboração de imagens relacionadas à SIG para utilizações diversas relacionadas à tomada de decisão, contando com uma definição de tarefa do usuário proposta para o desenvolvimento dessa investigação.

Como resultado, tem-se uma proposta de gramática de interação que conta com seis tipos distintos de toques necessários a execução integral das tarefas estipuladas para a IIT, de modo fundamentado na pesquisa apresentada.

O estudo também possibilitou uma melhor compreensão sobre interfaces naturais. A relação entre seu conceito e sua prática efetiva ainda carece de aprimoramento, pois o alcance de uma interação que seja efetivamente natural, requer recursos de maior complexidade, no que diz respeito ao uso das capacidades sensório motoras do homem.

Percebe-se, nesse contexto, que a questão de desenvolvimento da tecnologia vem tornando o conceito de interface natural cada vez mais uma realidade, mas que ainda permanece, com maior vigor, como potencialidade.

Por fim, entende-se que o presente trabalho cumpre seu objetivo e favorece estudos relacionados à interação de artefatos de interação natural, tanto em relação ao construto realizado como a partir de métodos e instrumentos utilizados.

\section{Bibliografia}

Android Developers. (S.1.): Android, 2013. Disponível em <http://developer.android.com/ index. html>. Acesso em: 17 jul. 2013.

BASTIEN, J. M. C.; SCAPIN, D. L. RT-0156 - Ergonomic criteria for the evaluation of humancomputer interfaces. Rapport technique de l'INRIA, (S.l.: S.n.),1993. Disponível em: <http://hal.inria. fr/docs/oo/o7/oo/12/PDF/RT-0156.pdf>. Acesso em: 07 mai. 2017.

BAXTER, M. Projeto de produto: guia prático para o desenvolvimento de novos produtos. São Paulo: Edgard Blücher, 1998.

CYBIS, W. A. Engenharia de usabilidade: uma abordagem ergonômica. Florianópolis: Labiutil, 2003.

DIX, A. et. al. Human-computer Interaction. 3rd ed. Essex, England: Pearson/Prentice-Hall, 2004. 
IOS Dev Center. (S.l.): Apple, 2012. Disponível em <http://developer.apple.com/devcenter/ios/ index.action>. Acesso em: 17 jul. 2013.

ISO 9241. 1993, Ergonomic requirements for office work with visual display terminals, Part 1 General Introduction. ISO

MICROSOFT Surface: Development Overview. (S.l.): Microsoft, 2013. Disponível em <http://msdn. microsoft.com/en-us/library/ ee786837(v=surface.10).aspx>. Acesso em: 17 jul. 2013.

NIELSEN, J.; MOLICH, R. Heuristic evaluation of user interfaces. Proceedings of the SIGCHI Conference on Human Factors in Computing Systems / CHI'9o. New York, USA: ACM, 1990. Disponível em: <http://doi.acm.org/10.1145/ 97243.97281>. Acesso em: o7 mai. 2017.

NICOLELIS, M. Muito além do nosso eu: a nova neurociência que une cérebro e máquinas e como ela muda nossas vidas. São Paulo: Companhia das Letras, 2011.

NORMAN, D. A. Natural user interfaces are not natural. Interactions, New York, USA, v. 17, issue 3, p. 6-10, May-June 2010.

OLIVEIRA, G. P. C.; SILVA, C. E. Multi-touch: uma abordagem experimental da evolução dos computadores. Ciência \& Consciência, (S.l.), v. 1, n. - , 2008. Disponível em: <http://www.revista. ulbrajp.edu.br/seer/inicia/ojs/viewarticle.php?id=1822>. Acesso em: 09 mai. 2017.

PASSOS, R. Design da informação: modelo para configuração de interfaces naturais. Tese de doutorado em Design. Universidade de Aveiro, 2014.

. O design da informação em interfaces de hipermídias. Dissertação (Mestrado em Design). Universidade Anhembi Morumbi: São Paulo, 2008a.

. Percursos do projeto de design. In: SILVA, Jofre. (Org.). Design, arte e tecnologia. São Paulo: Rosari, Universidade Anhembi Morumbi, PUC-Rio e Unesp-Bauru, 2008b, n. 4.

PREECE, J.; ROGERS, Y.; SHARP, H. Design de interação: além da interação homem-computador. Porto Alegre: Bookman, 2005.

SAFFER, D. Designing gestural interfaces: touchscreens and interactive devices. Sebastopol, CA: O’Reilly, 2009.

VILLAMOR, C.; WILLIS, D.; WROBLEWSKI, L. Touch gesture reference guide. San Francisco, USA: LukeW, 2010. Disponível em: <http://www.lukew.com/ff/entry.asp?1071>. Acesso em: 07 mai. 2017.

WINDOWS Touch: Developer Resource. [S.l.]: Microsoft, 2013. Disponível em <http://archive. msdn.microsoft.com/WindowsTouch>. Acesso em: 17 jul. 2013. 